\title{
NOUVELle
}

\section{À l'âge de 8 mois, les bébés ont déjà intégré la grammaire de leur langue maternelle}

Caterina Marino ${ }^{1}$, Carline Bernard ${ }^{2}$, Judit Gervain ${ }^{1}$

${ }^{1}$ Centre de neurosciences intégratives et cognition CNRS UMR 8002, 45 rue des Saints Pères, 75006 Paris, France. ${ }^{2}$ Centre hospitalier Charles Perrens, 121 rue de la Béchade, 33000 Bordeaux, France. cateemar@gmail.com carline.bernard@gmail.com judit.gervain@parisdescartes.fr
> L'apprentissage de l'ordre des mots est l'un des premiers exploits accomplis par les nourrissons au cours de l'acquisition du langage parlé. Différentes études ont montré que les enfants connaissent l'ordre correct de leur langue native quand ils commencent à produire leurs premières phrases, à un âge compris entre 2 et 3 ans [1]. Mais comment et quand les enfants commencent-ils à acquérir cet ordre des mots? Et quelles sont les caractéristiques de la langue qui les aident dans cet apprentissage?

Une caractéristique universelle du langage humain est la distinction et l'agencement entre les «mots de fonction » (les articles et autres déterminants, les pronoms, et les prépositions, tels que le, $i l$, en, sur, etc.), qui marquent la structure grammaticale, et des «mots de contenu » (les noms, les verbes, les adjectifs, et les adverbes, comme arc-en-ciel, manger, beau, simplement, etc.), qui portent le sens lexical. Pour les nourrissons, l'identification des mots de fonction constitue une première étape cruciale dans l'acquisition de la grammaire de leur langue native. Les mots de fonction sont très fréquents, tandis que les mots de contenu le sont moins. D'où l'hypothèse que les nourrissons s'appuieraient sur la fréquence d'occurrence des mots pour établir ces catégories lexicales de base [2-4]. Cependant, les preuves directes font toujours défaut. On ne sait pas exactement comment et à quel âge les nourrissons utilisent pour la première fois ces éléments de base du langage pour en extraire les modèles grammaticaux pertinents.
La majorité des langues naturelles sont configurées de deux manières possibles, selon que les mots de fonction précèdent ou suivent les mots de contenu. Ainsi, l'agencement de ces deux types de mots (qui forment ensemble l'unité syntaxique, ou syntagme) varie selon les langues. En français ou en anglais par exemple, les mots de fonction apparaissent en début de syntagme (e.g., à Paris, in London), alors qu'en japonais ou en basque, les mots de fonction apparaissent en fin de syntagme (e.g., Tokyo ni, garren atzean). Il a été proposé que les nourrissons utilisaient les mots de fonction du signal de parole comme point d'ancrage, codant la position des autres mots par rapport à eux $[5,6]$. Comme la différence de fréquence d'occurrence entre les mots de fonction et les mots de contenu est universelle d'une langue à l'autre, le mécanisme d'amorçage («bootstrapping ») fondé sur la fréquence de ces catégories lexicales pourrait fournir un outil puissant pour que les nourrissons puissent intégrer les règles de leur langue. Pour tester cette hypothèse, nous avons utilisé cette caractéristique distinctive des mots de fonction et des mots de contenu comme outil diagnostique.

De plus, les mots de contenu forment des classes ouvertes: nous ajoutons fréquemment de nouveaux mots à notre lexique (e.g., iPad, Brexit, etc.), tandis que les mots de fonction constituent des classes fermées, dans lesquelles de nouveaux éléments ne peuvent pas être ajoutés sans entraîner une modification radicale de la langue. Si les représentations linguistiques des nourrissons sont telles que les mots fréquents y constituent des classes fermées et les mots non fréquents des classes ouvertes, cela signifie qu'ils sont déjà sensibles à l'une des caractéristiques qui différencient les mots de fonction des mots de contenu.

Les enfants sont-ils déjà sensibles à cette information à l'âge de 8 mois ? Pour le savoir, nous avons utilisé, chez des enfants francophones de 7 à 9 mois, une tâche d'apprentissage d'une minilangue inventée. Les résultats de cette étude ont été publiés dans la revue Current Biology [7]. Nous avons ainsi familiarisé six groupes d'enfants avec la même langue artificielle, dans laquelle les mots fréquents, représentant les mots de fonctions de notre langue naturelle, alternaient strictement avec les mots non fréquents, représentant les mots de contenu (...gekafimugenafifogebi...), un paradigme précédemment utilisé par d'autres chercheurs [4, 8]. Ainsi, les bébés écoutaient un flux sonore dans cette mini-langue pendant 4 minutes, mais l'intensité sonore augmentait progressivement pendant les 15 premières secondes et diminuait pendant les 15 dernières secondes, de façon à rendre ambiguë la structure de la langue artificielle (la perception de l'agencement initial et final des mots de fonction par rapport aux mots de contenu devenait impossible). Nous avons réalisé six expériences, avec six groupes de bébés; ils ont tous été familiarisés avec la même minilangue, mais les items tests différaient dans chaque groupe, selon la nouveauté 
A

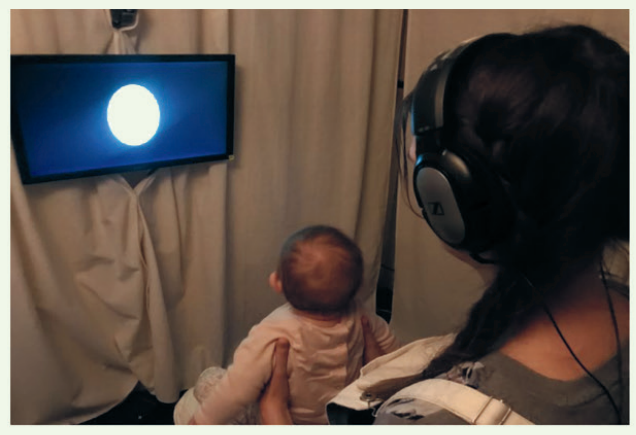

B

Phase de familiarisation

...АХВУАХХВУА...

$\{X ; Y\}:\{A ; B\}=1: 9$

$A:\{\mathrm{fi}\}$

$X:\{r u, p e, d u, b a, f o, d e, p a, r a$, to $\}$ $B:\{\mathrm{ge}\}$

$y:\{m u, r i, k u, b o, b i, d o, k a, n a, r o\}$

...gekafirugenafifogebi...
$F=$ fréquent

$I$ = infréquent

$\mathrm{N}=$ Nouveau

Expérience 1:

$$
\text { F-I-F-I }
$$

fifogebi
firugemu

gerifipe

gedofide

F-N-F-N

figogene

Expérience 2 :

fisegemo

gemafisho gefafoso

N-I-N-I

sefoshobi

Expérience 3 : $\quad$ serushomu

shorisepe

shodosede

I-I

Expérience 4 :

kuna

naku

$\mathbf{N}-$ I-N-I

sefoshobi

shorisepe

shodosede

I-N-I-N

bashobose

kasepasho

Expérience 6 : kusedusho

rashonase

40
Expérience 5 : $\quad$ serushomu

I-F-I-F
bagebofi
kafipage
kufiduge
ragenafi
N-F-N-F
tatifuge
nugetifi
shefivuge
vigegafi
I-N-I-N
bashobose
kasepasho
kusedusho
rashonase
N-N
tigo
goti
N-N-N-N
senushoti
segoshone
shogasevi
shomosefa
N-N-N-N
senushoti
segoshone
shogasevi
shomosefa
Temps (min)

Figure 1. Phases de familiarisation et de test. A. Exemple d'un enfant en train d'effectuer la tâche d'apprentissage de notre mini-langue inventée. B. Tâche de grammaire artificielle utilisée dans les six expériences.

«n'acceptent pas » de nouveaux mots fréquents, mais s'adaptent facilement aux nouveaux mots infréquents (Figure 2). Ces résultats viennent compléter les données de la littérature chez les nourrissons concernant d'autres caractéristiques distinctives de ces deux classes de mots, telles que leurs différentes formes phonologiques [9] et leurs différences fonctionnelles $[4,10,11]$. Ils apportent d'autres preuves de la capacité des nourrissons à distinguer ces deux classes lexicales, en montrant que ces derniers sont capables, à partir de la fréquence des mots dans une langue artificielle, d'extraire des informations importantes concernant 1) leur position: la langue française place les mots fréquents au début des syntagmes (e.g., à Paris, le chat, je vais...), alors que la langue japonaise les place en position finale (e.g., «Tokyo kara», que I'on peut traduire littéralement par «Tokyo de »), et 2) leur fonction: de nouveaux mots de contenu apparaissent dans digm, HPP) fondée sur une mesure des temps de regard relatifs aux items-tests (Figure IA).

Les résultats de cette expérience ont montré, d'une part, que les bébés de 8 mois ont une préférence (temps de regard plus longs) pour les phrases comportant un ordre des mots typique du français, et d'autre part, qu'ils peuvent utiliser la fréquence des mots pour identifier la catégorie à laquelle appartient un mot spécifique. En effet, ils la langue presque chaque jour (e.g., iPhone, courriel, etc.), tandis qu'il n'est pas possible d'y ajouter un nouveau mot de fonction (impossible, par exemple, d'inventer une nouvelle préposition ou un nouveau déterminant).

Nous avons donc montré que les bébés ont des connaissances des bases de la grammaire de leur langue native plusieurs mois avant de produire leurs premiers mots. Les bébés francophones de 8 mois savent déjà que les mots 


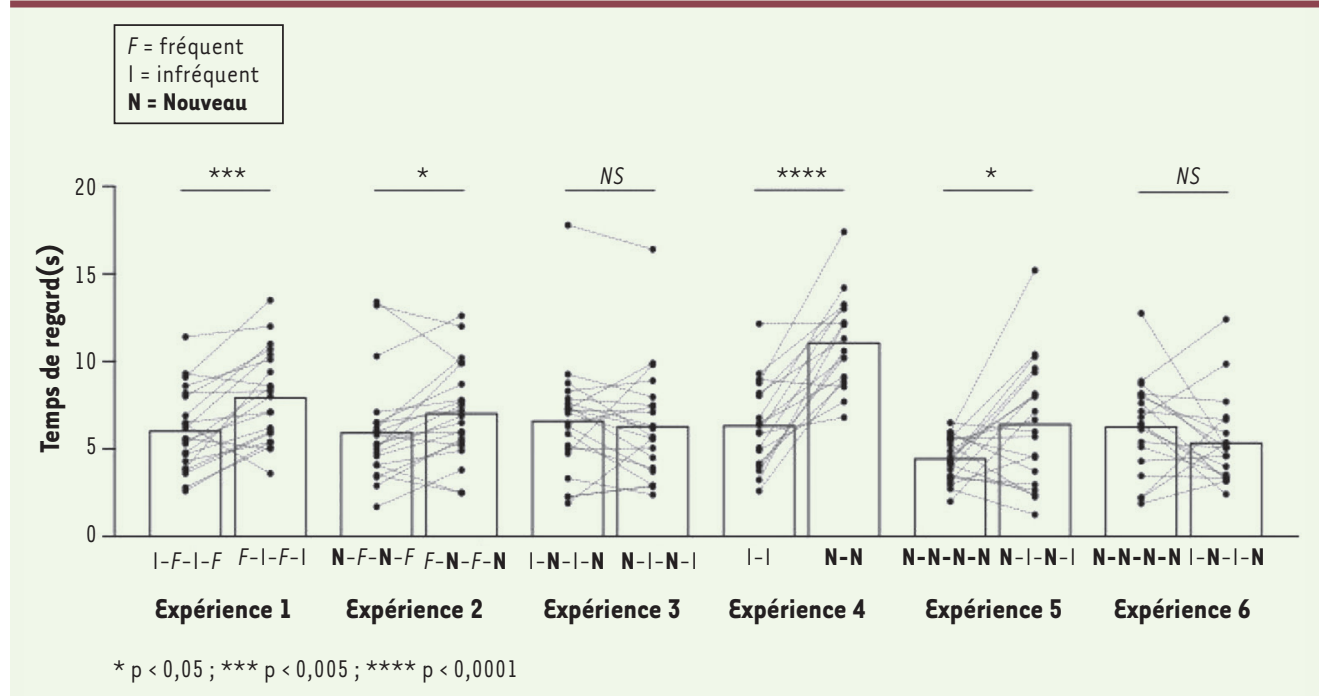

Figure 2. Temps de regard (looking time), en secondes (s), obtenus selon l'ordre des phrases de la phase de test, pour les six expériences. Les barres horizontales représentent la moyenne du groupe; les points connectés représentent les résultats individuels pour chaque bébé participant. NS : non significatif.

fréquents sont des mots grammaticaux qui apparaissent en début de syntagme en français, et qui ne peuvent pas être remplacés par de nouveaux éléments, tandis qu'ils acceptent volontiers un nouveau mot en tant que mot de contenu. L'apprentissage de la grammaire commence donc plus tôt que ce que I'on croyait, parallèlement à l'apprentissage des sons et des mots, bien avant les premières productions vocales de mots par l'enfant. $\diamond$

Word frequency is a cue to lexical category for 8-month-old infants

\section{LIENS D'INTÉRÊT}

Les auteures déclarent n'avoir aucun lien d'intérêt concernant les données publiées dans cet article.

\section{RéFÉRENCES}

1. Brown RW. A first language: the early stages. Cambridge : Harvard University Press, 1973 : 438 p.

2. Braine MD. Learning the positions of words relative to a marker element.J Exp Psychol 1966 ; 72 : 532-40.

3. Morgan JM, Newport EL. The role of constituent structure in the induction of an artificial language. J Verbal Learn Verbal Behav $1981 ; 20$ : 67-85.

4. Gervain J, Nespor M, Mazuka R, et al. Bootstrapping word order in prelexical infants: a Japanese-Italian cross- linguistic study. Cognit Psychol 2008 ; 57 : 56-74

5. Valian V, Levitt A. Prosody and adults' learning of syntactic structure. J Mem Lang 1996 ; 35 : 497-516.
6. Morgan JL, Meier RP, Newport EL. Structural packaging in the input to language learning: contributions of prosodic and morphological marking of phrases to the acquisition of language. Cognit Psychol $1987 ; 19$ 498-550.

7. Marino C, Bernard C, Gervain J. Word frequency is a cue to lexical category for 8-month-old infants. Curr Biol 2020 ; 30 : 1380-6.

8. Bernard C, Gervain J. Prosodic cues to word order: what level of representation? Front Psychol 2012; $3: 451$.

9. Shi R, Werker JF, Morgan JL. Newborn infants' sensitivity to perceptual cues to lexical and grammatical words. Cognition $1999 ; 72$ : B11-21.

10. Hochmann JR, Endress AD, Mehler J. Word frequency as a cue for identifying function words in infancy. Cognition 2010 ; 115 : 444-57.

11. Hochmann JR. Word frequency, function words and the second gavagai problem. Cognition 2013 ; 128 : 13-25.

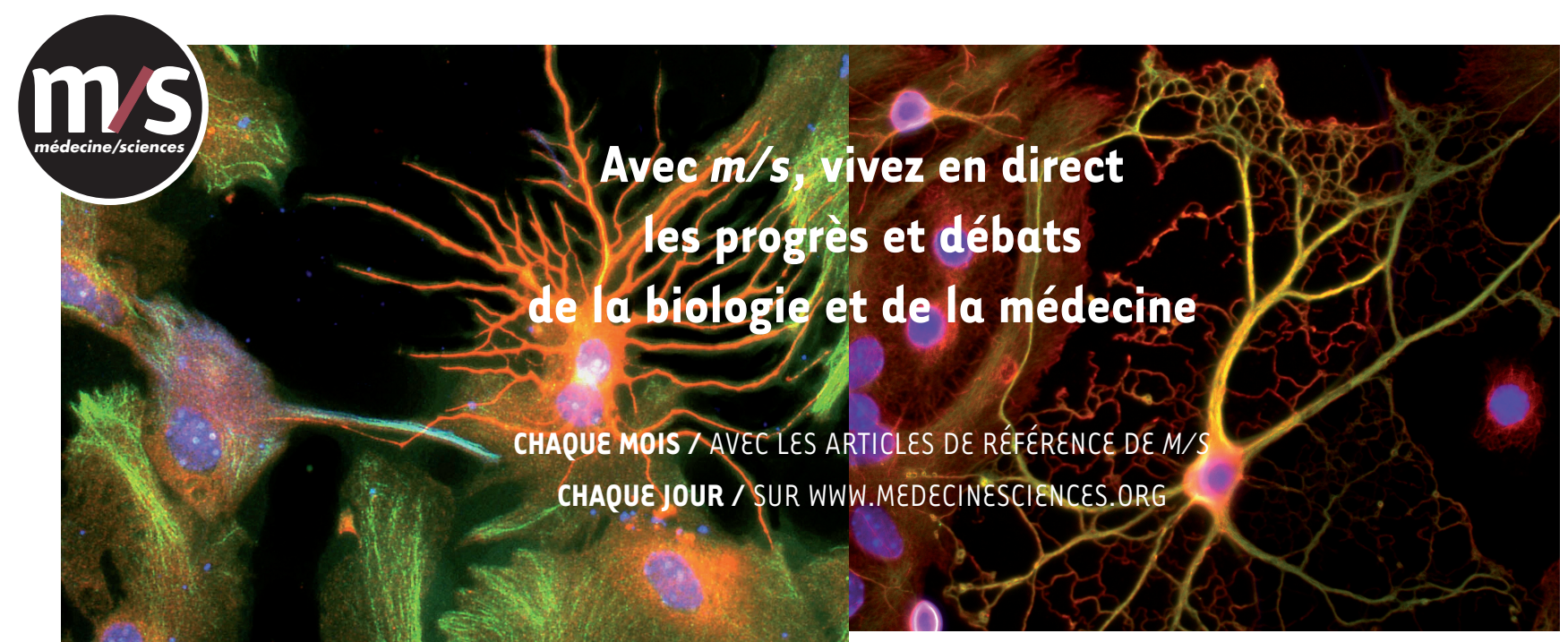

Abonnez-vous sur

WWW.medecinesciences.org 